\title{
La Anatomía Patológica en las Redes Sociales. \\ Campaña de Reclutamiento.
}

Eduardo Alcaraz Mateos, Servicio de Anatomía Patológica, Hospital Universitario Morales Meseguer, Murcia, España.

Laura Guerra Pastrián, Servicio de Anatomía Patológica, Hospital Universitario La Paz, Madrid, España.

Lara Pijuan Andújar, Servicio de Anatomía Patológica, Hospital del Mar, Barcelona, España.

Laura López Solache, Servicio de Anatomía Patológica, Complejo Hospitalario Universitario A Coruña, España.

Adriana Zucchiatti, Servicio de Anatomía Patológica, Hospital Vall d’Hebron, Barcelona, España.

Rubén García Ángel, Servicio de Anatomía Patológica, Hospital General de Albacete, España.

Juan Daniel Prieto Cuadra, Servicio de Anatomía Patológica, Hospital Clínico Universitario Virgen de la Victoria, Málaga, España.

Tania Labiano Miravalles, Servicio de Anatomía Patológica, Complejo Hospitalario de Navarra, España.

Rita Carvalho, Servicio de Patología, Central Lisbon Hospital Center, Portugal.

Jerad M. Gardner, Departments of Pathology and Dermatology, University of Arkansas for Medical Sciences, Little Rock, Arkansas, USA.

Cristina Terrádez. Sociedad Española de Anatomía Patológica, Madrid, España.

Enrique de Álava, Servicio de Anatomía Patológica, Hospital Universitario Virgen del Rocío, Sevilla, España.

Contacto:

Eduardo Alcaraz Mateos

Teléfono: 699297783

Email: edusqo@hotmail.com 


\section{La Anatomía Patológica en las Redes Sociales. \\ Campaña de Reclutamiento.}

\section{INTRODUCCIÓN}

La Anatomía Patológica sigue siendo desconocida, no sólo para el público en general sino también para nuestros propios compañeros. El uso generalizado de las redes sociales permite difundir nuestra labor. Por ello, se planteó generar un grupo de trabajo.

\section{MATERIAL Y MÉTODOS}

Se emitió una llamada a los socios de la Sociedad Española de Anatomía Patológica a través de su Vocalía de Comunicación y Proyección Social titulada \#IWantYouForSEAP, para conformar una red de trabajo en la red social Twitter. El periodo de reclutamiento fue de un mes (agosto, 2016). Se registró la actividad desarrollada durante el XVIII Congreso de la SEAP-IAP de 2017 utilizando las herramientas analíticas Symplur y Tweet Binder.

\section{RESULTADOS}

Se recibieron 32 solicitudes (28 patólogos, 2 histotécnicos, 1 administrativo) con una dispersión geográfica dentro del territorio español que incluyó a participantes de 14 de las 17 comunidades autónomas, perteneciendo a 22 ciudades y 25 centros distintos. La actividad en relación al hashtag \#SEAP2017V utilizado en el congreso incluyó 685 participantes, 6704 tweets y 8.837 .435 impresiones. 28 de los 32 reclutados por la campaña \#IWantYouForSEAP participaron, contribuyendo con 2410 tweets, y generando 2.090 .423 impresiones (36\% y $24 \%$ del total, respectivamente).

\section{CONCLUSIONES}

Es posible promover y motivar el trabajo en grupo dentro de nuestra disciplina a través de las redes sociales.

Se trata de una fase inicial, pero los resultados experimentados son alentadores y existen grandes expectativas por parte de los participantes.

Un uso adecuado de las redes sociales podría acercar la figura del patólogo a la sociedad. 


\section{Pathology in Social Media Networks. \\ Recruitment Campaign.}

\section{INTRODUCTION}

Pathology continues to be unknown, not only to the general public but also to our physician colleagues. Nowadays, the widespread use of social media networks allows us to present and spread our occupation. Therefore, it was proposed to create a working group.

\section{MATERIAL AND METHODS}

The members of the Spanish Society of Pathology were contacted through its Communication and Social Projection Subcommittee to engage in the campaign \#IWantYouForSEAP, to form a network on Twitter. The recruitment period was one month (August, 2016). The activity developed during the XVIII Congress of the SEAP-IAP was registered using the analytical tools Symplur and Tweet Binder.

\section{RESULTS}

32 applications (28 pathologists, 2 histotechnicians, 1 administrative) were received with a geographic dispersion within the Spanish territory that included participants from 14 of the 17 autonomous communities, belonging to 22 cities and 25 different centers. The activity in relation to the hashtag \# SEAP2017V used in the congress included 685 participants with 6704 tweets and 8,837,435 impressions. 28 of the 32 recruited by the \#IWantYouForSEAP campaign participated, contributing with 2410 tweets, and generating 2,090,423 impressions ( $36 \%$ and $24 \%$ of the total, respectively).

\section{CONCLUSIONS}

It is possible to promote and motivate teamwork within our discipline through social media networks.

This is an initial inquiry, but the results are encouraging and there are high expectations on behalf of the participants.

Proper use of social media networks could help to closet the gap between pathologists and society. 


\section{INTRODUCCIÓN}

La figura del patólogo sigue siendo desconocida, no sólo para el público en general sino también para nuestros propios compañeros de profesión y podemos ser considerados incluso como "el doctor invisible", como ya hizo mención el Dr. López en The Pathologist". La información vertida por los medios de comunicación habitualmente es insuficiente e incluso resulta errónea en muchas ocasiones.

El uso generalizado actual de las redes sociales (RRSS) permite presentar y difundir la actividad científica o asistencial de los profesionales de la salud hacia la sociedad y hacia los pacientes, que son el fin último de nuestro trabajo. Esto contribuiría a la denominada health literacy ${ }^{2-5}$, que se podría traducir como la "alfabetización en salud" y que se podría definir como "la capacidad para obtener, interpretar y comprender información médica con el fin de tomar las decisiones de salud apropiadas y seguir las instrucciones de los tratamientos"; en resumen, que el paciente sepa de qué habla su médico.

Al mismo tiempo, estas herramientas web 2.0 de interacción se han demostrado útiles tanto como soporte informativo de revistas científicas ${ }^{6}$ y otros medios, como para la comunicación entre nosotros mismos (networking, manejo clínico, investigación o incluso constituyendo clubs de lectura crítica, i.e. journal clubs) ${ }^{7-13}$ o a nivel docente 0 formativo con estudiantes de medicina o residentes ${ }^{14-15}$.

Las redes sociales más utilizadas en la actualidad por los profesionales de la salud, son Facebook (Palo Alto, CA, EEUU, fundada en 2004) y Twitter (San Francisco, CA, EEUU, fundada en 2006), con 1968 y 328 millones de usuarios, respectivamente (fuente: www.statista.com, consultado en junio de 2017).

Por todo esto, se planteó generar un grupo de difusión de anatomía patológica con el cometido de hacer nuestra profesión más visible. Seleccionamos Twitter como plataforma por sus características de microblogging limitado a 140 caracteres y por considerarlo un medio más adecuado para dicho propósito. Además, dicha campaña incluiría entre sus fines la promoción de la actividad científica a desarrollar durante el XXVIII Congreso de la Sociedad Española de Anatomía Patológica (SEAP-IAP) de Valencia en 2017. En este sentido, son numerosas las experiencias en relación al uso "en vivo" de las redes sociales en congresos científico-médicos como ASCO (American Society of Clinical Oncolgy) ${ }^{16} 0$ USCAP $^{17}$ (United States \& Canadian Academy of Pathology), entre otras ${ }^{17-21}$.

\section{MATERIAL Y MÉTODOS}

Se emitió una llamada a los miembros de la Sociedad Española de Anatomía Patológica (SEAP-IAP) a través de su Vocalía de Comunicación y Proyección Social. Se realizó el envío de un formulario vía email para la adhesión a una red de trabajo en la red social Twitter. La campaña fue titulada \#IWantYouForSEAP, y en ella, se asoció la figura del histólogo y patólogo premio Nobel español Don Santiago Ramón y Cajal, junto al conocido eslogan "I want you" de la pancarta de 1917, creada por James Montgomery Flagg para el 
alistamiento en la I Guerra Mundial (figura 1). Se adjuntó información acerca de dicha plataforma y de las nociones básicas para su uso de un modo profesional ${ }^{23-25}$, incluyendo recomendaciones de cuentas y usuarios influencers (@), así como etiquetas o hashtags (\#). El periodo de reclutamiento se llevó a cabo durante el mes de agosto de 2016 y se obsequiaría a los primeros solicitantes con un "kit de supervivencia" que incluyó un cargador de móvil, una memoria USB, una batería externa, una chapa acreditativa, entre otros ítems (figura 1').

En un segundo tiempo, teniendo como objetivo conocer la actividad desarrollada durante el XXVIII Congreso de la SEAP-IAP, se obtuvieron los datos del hashtag elegido como identificativo del congreso, \#SEAP2017V, en Twitter, utilizando las herramientas analíticas de Symplur (Los Angeles, CA, EEUU, fundada en 2011 y con sitio web: www.symplur.com) y de Tweet Binder (Pamplona, España, fundada en 2012 y con sitio web: www.tweetbinder.com), ambas centradas en el análisis de datos de dicha plataforma. Se consideró un periodo de tiempo de 13 días en torno al congreso, desde el 18 de mayo de 2017 hasta el 31 del mismo mes, un rango de fechas similar al planteado en la publicación de Cohen y col. ${ }^{17}$ en relación al Congreso de la Academia Americana y Canadiense de Patología (USCAP) de 2015 (\#USCAP2015).

\section{RESULTADOS}

Un total de 32 solicitudes fueron recibidas, incluyendo 17 mujeres y 15 hombres, con una media de edad de 38.6 años (rango 23-59, desviación estándar 10.8), siendo 28 de los solicitantes patólogos (incluyendo 6 residentes, 4 de segundo año y 2 de tercer año), 2 histotécnicos, 1 biólogo y 1 administrativo (figura 2). Existió una dispersión geográfica notable dentro del territorio español, incluyendo a participantes de 14 de las 17 comunidades autónomas, perteneciendo a 22 ciudades y 25 centros distintos. Sólo 3 de los solicitantes no habían tenido contacto previo con Twitter. Existió unanimidad en las cuestiones incluidas en el formulario en relación a la utilidad profesional de las redes sociales y a la mayor visibilidad del patólogo a través de un buen uso de las mismas.

En relación a los resultados del Congreso bienal celebrado en Valencia, con un número aproximado de 1200 asistentes, y a su etiqueta \#SEAP2017V, se obtuvieron un total de 8.837.435 impresiones o impacto potencial (número de veces que los usuarios de Twitter han podido ver una determinada publicación con dicha etiqueta), 781.724 usuarios de alcance potencial (número de usuarios distintos que pueden haberla visto), 6704 tweets y retweets (mensajes), y de éstos, 1105 originales (16,5\%), con una distribución por días según la figura 3 , a una media de 20 tweets/hora. Igualmente, se registraron 685 participantes (usuarios que twittean 0 interaccionan), con una media de 10 tweets por participante: 1 tweet (52\%), entre 2 y 6 tweets $(27 \%)$ y más de 6 tweets (21\%). Los usuarios más influyentes o influencers se muestran en la figura 4. 28 de los 32 reclutados por la campaña \#IWantYouForSEAP participaron en \#SEAP2017V, contribuyendo con 
2410 tweets, de los cuales, 351 eran originales, con un total de 2.090 .423 impresiones, y representando el $36 \%, 31,8 \%$ y $24 \%$ del total, respectivamente.

Con ciertas limitaciones, a consecuencia de la dificultad en algunos casos, se ha podido cuantificar el número de tweets por contenido, según sea únicamente de carácter científico: publicaciones de comunicaciones o pósteres con fotografías y contenido científico en el cuerpo de la publicación (495 tweets, $45 \%$ del total); relacionado con las actividades del congreso: actos de inauguración y clausura, notas informativas, premios, exposición de las obras de arte del I Concurso de Artes Plásticas \#SEAPArt, marcha divulgativa por el cauce del río Turia, firma del libro "Ramón y Cajal: Una vida al microscopio" de Jordi Bayarri, obsequiado a los asistentes, publicaciones en otros medios de comunicación y otros (183 tweets, $16,6 \%$ del total); y exclusivamente considerados sociales: opiniones personales, fotos de grupo, autofotos o selfies y otros aspectos lúdicos (427 tweets, 38,6\% del total). Las empresas o industria colaboradora en el congreso participó con menos del $0,5 \%$ de la actividad (33 tweets, 32 tweets originales y 13.227 impresiones), una cifra mucho menor que en otros congresos, donde alcanza el $2-5 \%^{16,20}$. No se observaron publicaciones de contenido ajeno al congreso ni ofensivas.

La procedencia de los participantes, en aquellos en los que se pudo comprobar (403 usuarios), correspondió a: España (198, 49,1\%), Estados Unidos y Canadá (70, 17,4\%), Latinoamérica $(65,16,1 \%)$, Asia $(30,7,4 \%)$, resto de Europa $(29,7,2 \%)$, África $(8,2 \%)$ y Australia (3, 0,7\%) (figura 5).

Por último, las etiquetas más prevalentes, junto a \#SEAP2017V, fueron: \#Pathology, \#PulmPath, \#IWantYouForSEAP, \#Pathologists, \#Valencia, \#PDL1, \#SoMe, \#Pathologist, \#llooklikeapathologist.

\section{DISCUSIÓN}

Es indudable el continuo crecimiento que en los últimos años está teniendo el uso profesional de las redes sociales, y así está quedando reflejado en las bases de datos científicas, con keywords como Twitter, Social Media o Facebook, en los ámbitos docente, asistencial e investigador, pero fundamentalmente repercutiendo en la visibilidad de las distintas disciplinas a través de sus congresos y revistas científicas. Estas herramientas, basadas en la teoría de los "seis grados de separación" plasmada por Frigyes Karinthy en su relato Chains de 1929, nos permiten a los patólogos, un colectivo minoritario dentro del ámbito sanitario trabajar nuestra visibilidad y tratar de recuperar la influencia que en otro tiempo tuvimos. Sin embargo, sigue existiendo cierta reticencia a su uso con finalidad profesional por motivos varios, tales como el desconocimiento, la falta de tiempo, o el temor a perjudicarse la propia imagen ${ }^{25}$. Esto, no obstante, puede solventarse con una formación adecuada basada en recomendaciones o guías de utilización elaboradas por las propias sociedades científicas ${ }^{26-28}$. 
Lo que hemos podido observar con la campaña de reclutamiento es que resulta posible promover y motivar el trabajo en grupo dentro de nuestra disciplina a través de las redes sociales, consiguiendo una rápida respuesta en poco tiempo gracias a las nuevas tecnologías (email, web, y las propias redes sociales). De hecho, tan sólo transcurrido un mes tras el congreso, el número de patólogos y otros profesionales españoles relacionados (técnicos, biólogos) con cuenta en Twitter ha superado la centena (129, consultado el 30 de Junio de 2017).

Se trata de la primera vez que se organiza y promueve el uso de las redes sociales en un congreso de la SEAP-IAP y vemos una actividad similar, e incluso superior, a la que se desarrolló en \#USCAP2015 cuando promovieron una iniciativa similar, teniendo en cuenta que el número de asistentes a USCAP es en torno a los 4000, frente a los 1200 de la SEAP-IAP, podemos considerarlo un éxito. La planificación y organización previa son grandes responsables del resultado obtenido, así como la participación de usuarios influencers, como el doctor Jerad Gardner @JMGardnerMD, con más de 10000 seguidores (o 10K), que consiguió de manera individual acaparar el $23 \%$ de las impresiones generadas durante el congreso. Los datos obtenidos acerca de la procedencia de los participantes de \#SEAP2017V evidencian una marcada proyección internacional del congreso, pese a tratarse de un congreso en español.

Encontramos ciertas limitaciones a la hora de cuantificar la actividad desarrollada en otros congresos para su comparación con nuestra experiencia. Por un lado, el rango de fechas es heterogéneo, desde sólo los días del evento a un rango mayor, como el que hemos planteado en nuestro estudio. Por otra parte, no todos los estudios contemplan los mismos ítems y por ejemplo, el número de tweets originales y su estudio y categorización según tipo o contenido (científico, social, industria), no queda reflejado. Nuestros datos manifiestan un porcentaje mejorable de tweets originales (16,5\%), así como de contenidos científicos (45\%), si bien no podemos obviar que se trata de una red social y que, por tanto, tanto las interacciones como las actividades sociales forman parte de la misma.

Se evidencia, por otra parte, que el "tweeting the meeting" o live-tweeting es creciente en USCAP, ASCO, y en otras especialidades como Urología, Oftalmología o Cirugía y que algunas sociedades científicas promocionan dicha actividad entre los asistentes a sus congresos a través de newletters, charlas previas a sesiones plenarias, posibilidad de añadir el usuario al badge, cursos precongreso y talleres, etc ${ }^{20}$.

Esta experiencia constituye una fase inicial y existen grandes expectativas de colaboración que incluyen los club de lectura crítica (Pathology Journal Clubs o \#PathJC) ${ }^{13}$, así como otros proyectos particulares que están viendo facilitada su difusión a consecuencia de las redes de patólogos nacionales e internacionales configuradas. Igualmente, se ha descrito la influencia en el factor de impacto de las revistas científicas la presencia o no de las mismas en las redes sociales ${ }^{6,29}$, por lo que la promoción de la Revista Española de Patología @REP_Patología es otro punto a trabajar. No debemos olvidar el trabajo que hay que realizar hacia nuestros compañeros de profesión, hacia el paciente y hacia los estudiantes para que sean capaces de descubrir la excelencia de esta disciplina. 
Por todo lo aquí descrito y por la posibilidad de acercar la figura del patólogo a la sociedad, animamos a todos los profesionales a formar parte de esta iniciativa.

\section{AGRADECIMIENTOS}

A la junta directiva, por facilitar esta propuesta y a todos los twitteros que han formado parte de la misma con su tiempo y dedicación.

1. JI López. "The invisible doctor". The Pathologist, 9, 46-48 (2015).

2. K. Hoedebecke, L. Beaman, J Mugambi, S. Shah, M. Mohasseb, C. Vettel et al. Health care and social media: What patients really understand. F1000Res. 2017 Feb 8;6:118.

3. J Cole, C Watkins, D Kleine. Health Advice from Internet Discussion Forums: How Bad Is Dangerous? J Med Internet Res. 2016 Jan 6;18(1):e4.

4. M Lepe, JM Gardner. Fine social aspiration: Twitter as a voice for cytopatholy. Diagn Cytopathol. 2017 Apr 2. doi: 10.1002/dc.23713. [Epub ahead of print].

5. A. Gough, RF. Hunter, O. Ajao, A. Jurek, G. McKeown, J Hong, et al. Tweet for behavior change: Using social media for the dissemination of public health Messages. JMIR Public Health Surveill. 2017 Mar 23;3(1):e14.

6. BS. Kelly, CE. Redmond, GJ. Nason, GM. Healy, NA. Horgan, EJ. Heffernan. The Use of Twitter by radiology journals: An analysis of Twitter activity and impact factor. J Am Coll Radiol. 2016 Nov;13(11):1391-1396.

7. MJ Markham, D Gentile, DL Graham. Social media for networking, profesional development, and patient engagement. Am Soc Clin Oncol Educ Book. 2017;37:782-787.

8. MY Fuller, TC Allen. Let's have a tweetup: The case for using Twitter professionally. Arch Pathol Lab Med. 2016 Sep;140(9):956-7.

9. L. Sinnenberg, AM. Buttenheim, K. Padrez, C. Mancheno, L. Ungar, RM. Merchant. Twitter as a Tool for Health Research: A Systematic Review. Am J Public Health. 2017 Jan;107(1):143.

10. KM Sternberg, SL Loeb, D Canes, L Donnelly, MH Tsai. The use of Twitter to facilitate sharing of clinical expertise in urology. J Am Med Inform Assoc. 2017 Jun 7. doi: 10.1093/jamia/ocx055. [Epub ahead of print].

11. Al Gardhouse, L Budd, SYC Yang, CL Wong. \#GeriMedJC: The Twitter Complement to the Traditional-Format Geriatric Medicine Journal Club. J Am Geriatr Soc. 2017 Jun;65(6):1347-1351. 
12. CE. Bayne, D. Cardnoa-Grau, MH. Hsieh. Introducing the Pediatric Urology Journal Club on Twitter. J Pediatr Urol. 2017 Feb;13(1):2-3.

13. M. Fuller, JM. Gardner, GM. Crane, SR. Williamson, S. Chiosea, CA. Arnold, et al. \#PathJC: The Founding and Success of the First Twitter Pathology Journal Club. Mod Pathol 2017 Vol 30:S2, 140A.

14. B. Diug, E. Kendal,D llic. Evaluating the use of twitter as a tool to increase engagement in medical education. Educ Health (Abingdon). 2016 Sep-Dec;29(3):223-230.

15. M. Sterling, P. Leung, D. Wright, TF. Bishop. The Use of Social Media in Graduate Medical Education: A Systematic Review. Acad Med. 2017 Feb 21. doi: 10.1097/ACM.0000000000001617. [Epub ahead of print]

16. N. Pemmaraju, MA. Thompson, RA.Mesa, T. Desai. Analysis of the use and impact of Twitter during American Society of Clinical Oncology annual meetings from 2011 to 2016: Focus on advanced metrics and user trends. J Oncol Pract. 2017 May 17:JOP2017021634. doi: 10.1200/JOP.2017.021634. [Epub ahead of print].

17. D. Cohen, TC. Allen, S. Balci, PT. Cagle, J. Teruya-Feldstein, SW. Fine, et al. \#InSituPathologists: how the \#USCAP2015 meeting went viral on Twitter and founded the social media movement for the United States and Canadian Academy of Pathology. Mod Pathol. 2017 Feb;30(2):160-168. doi: 10.1038/modpathol.2016.223. Epub 2017 Jan 13.

18. C. Udovicich, A. Barberi, K. Perera. Tweeting the meeting: A comparative analysis of an Australian emergency medicine conference over four years. J Emerg Trauma Shock. 2016 Jan-Mar;9(1):28-31.

19. SM. Christiansen, TA. Oetting, NL. Herz, JC. Law, CP. O'Brien, PS. Patel, et al.Twitter at the 2014 and 2015 Annual Meetings of the American Academy of Ophthalmology. Ophthalmology. 2016 Aug;123(8):1835-7.

20. DJ Attai, DM Radford, MS Cowher. Tweeting the meeting: Twitter use at the American Society of Breast Surgeons annual Meeting 2013-2016. Ann Surg Oncol. 2016 Oct;23(10):3418-22.

21. J. Campagne, J. Galland, O. Mangin. Use of Twitter in a congress: First experience for French internal medicine. Med Interne. $2016 \mathrm{Jul} ; 37(7)$ :497-501.

22. J. Gómez-Rivas, ME. Rodríguez-Socarrás, L. Tortolero-Blanco, M. García-Sanz, M. Alvarez-Maestro, MJ. Ribal, et al. Influencia de las redes sociales en congresos de sociedades y asociaciones urológicas: Resultados del 81ํㅡㄹ Congreso Nacional de la Asociación Española de Urología. Actas Urol Esp. 2017 Apr;41(3):181-187.

23. S. Ekins, EO. Perlstein. Ten simple rules of live tweeting at scientific conferences. Comput Biol. 2014 Aug; 10(8): e1003789. 
24. E. Madrigal, XS. Jiang, S. Roy-Chowdhuri. The professional Twitter account: creation, proper maintenance, and continuous successful operation. Diagn Cytopathol. 2017 Jul;45(7):621-628.

25. XS. Jiang, E. Madrigal, S.Roy-Chowdhuri. A Twitter primer: Dos and don'ts for cytopathologists. Diagn Cytopathol. 2017 Jul;45(7):577-579.

26. S. Patel, CM. Hawkins, JV. Rawson, JK. Hoang. Professional Social Networking in Radiology: Who Is There and What Are They Doing? Acad Radiol. 2017 May;24(5):574579.

27. AMA policy: professionalism in the use of social media. American Medical Association. https://www.ama-assn.org/sites/default/files/media-browser/public/about-professionalismuse-social-media.pdf. Consultado en Junio de 2017.

28. DG. Murphy, S. Loeb, MY. Basto, B. Challacombe, QD. Trinh, M. Leveridge, et al. Engaging responsibly with social media: the BJUI guidelines. BJU Int. 2014 Jul;114(1):9-11.

29. F. O'Kelly, GJ. Nason, RP. Manecksha, S. Cascio, FJ.Quinn, M. Leonard et al. The effect of social media (\#SoMe) on journal impact factor and parental awareness in paediatrica urology. J Pediatr Urol. 2017 Apr 21. pii: S1477-5131(17) 30169-9.

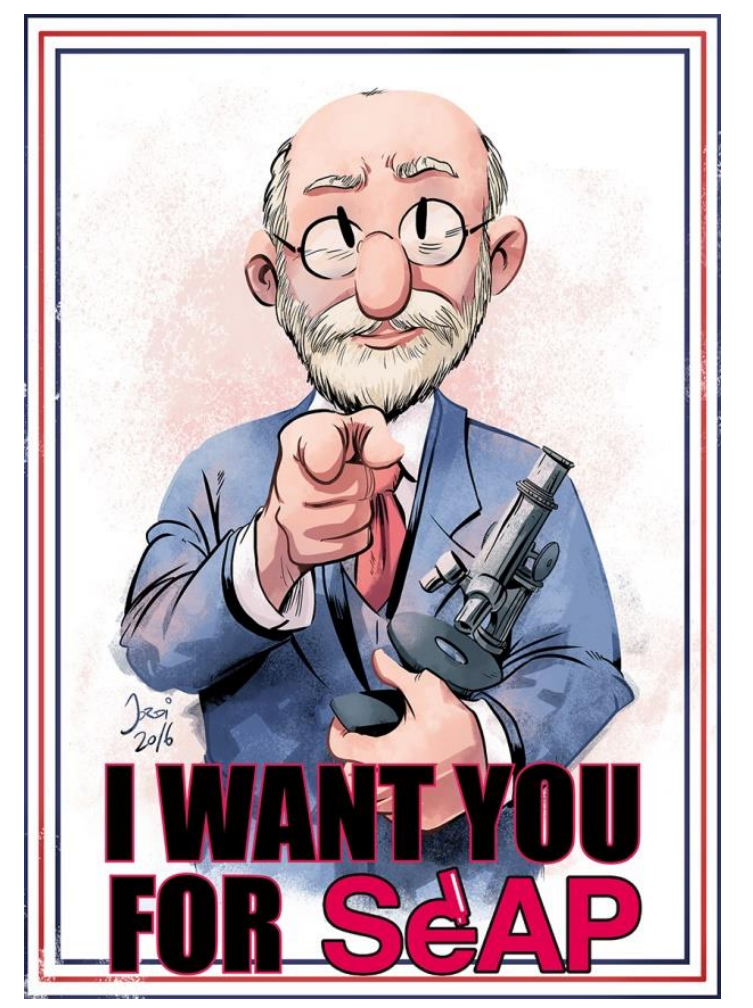

Figura 1. 


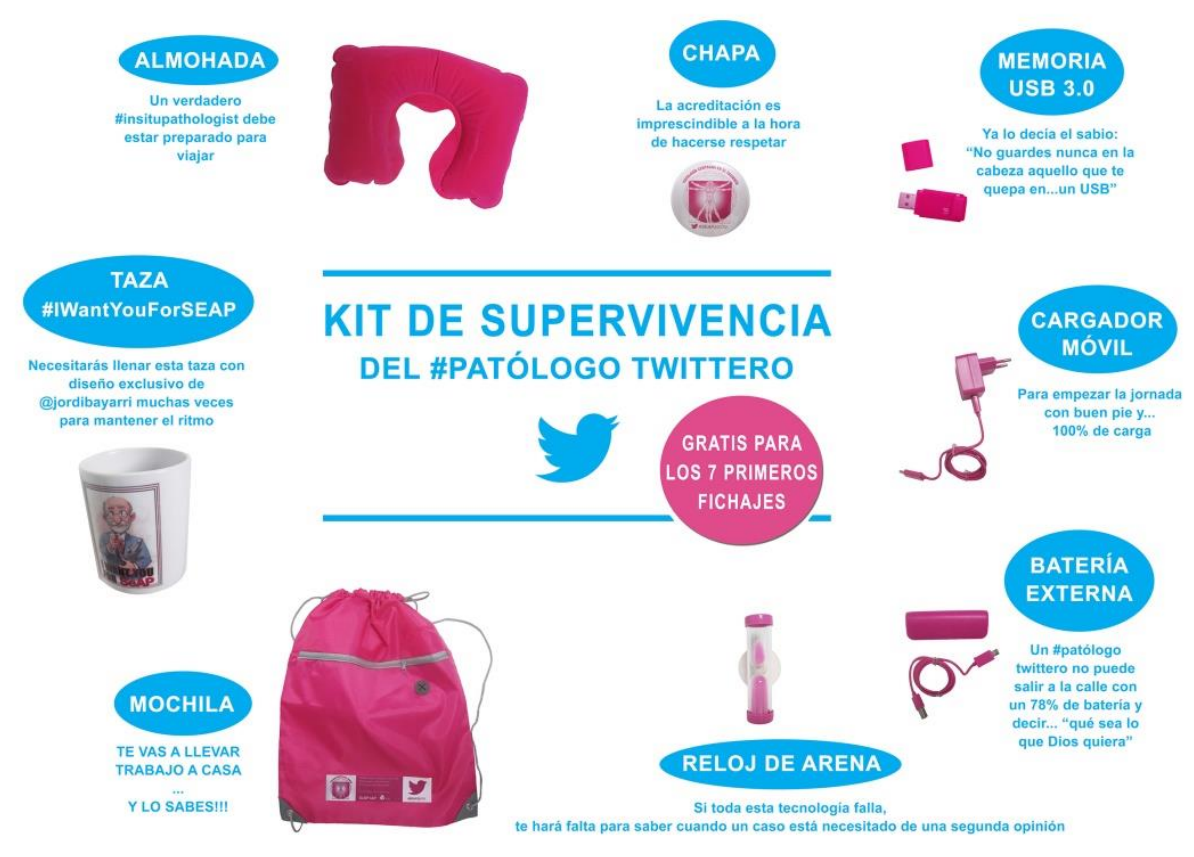

Figura 2.

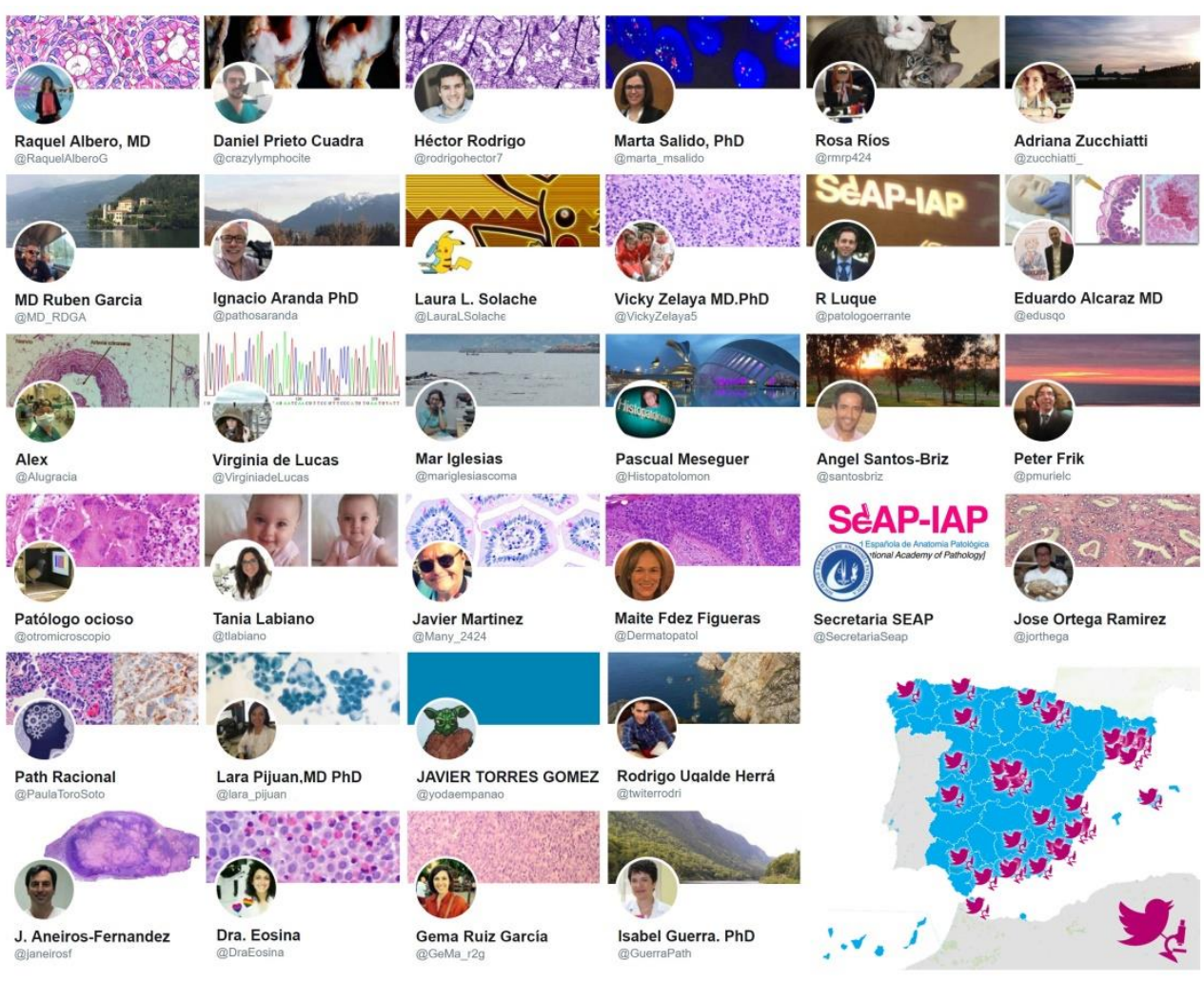

Figura 3. 


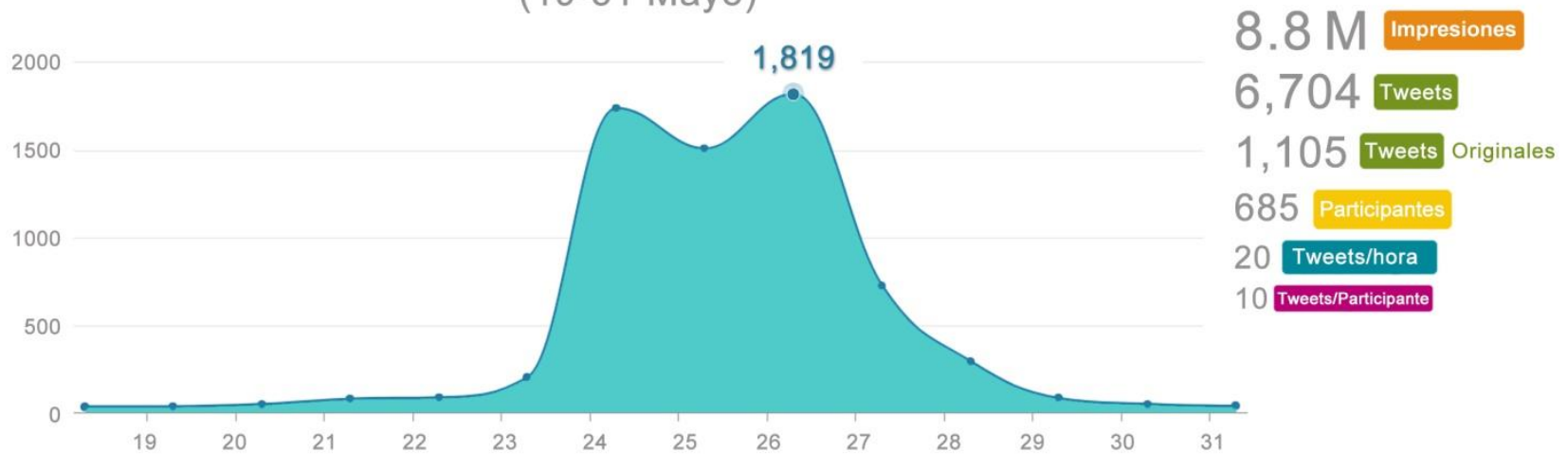

Figura 4.

Top 10 - Tweets originales

\begin{tabular}{|c|c|c|}
\hline \multicolumn{2}{|c|}{ articipante } & Tweets \\
\hline & $\begin{array}{l}\text { Jerad Gardner, MD } \\
\text { @JMGardnerMD }\end{array}$ & 83 \\
\hline & $\begin{array}{l}\text { Xelo Gimeno Borràs } \\
\text { @xelogimeno }\end{array}$ & 57 \\
\hline & $\begin{array}{l}\text { Eduardo Alcaraz MD } \\
\text { @edusqo }\end{array}$ & 55 \\
\hline & $\begin{array}{l}\text { Lara Pijuan,MD PhD } \\
\text { @lara_pijuan }\end{array}$ & 46 \\
\hline & $\begin{array}{l}\text { Stefania Landolfi } \\
\text { @steland011 }\end{array}$ & 44 \\
\hline & $\begin{array}{l}\text { SEAP-IAP } \\
\text { @SEAP_IAP }\end{array}$ & 36 \\
\hline & $\begin{array}{l}\text { amplia-mente } \\
\text { @amplia2mente }\end{array}$ & 31 \\
\hline & $\begin{array}{l}\text { Nacho Franco Rubio } \\
\text { @nfrancopath }\end{array}$ & 30 \\
\hline & $\begin{array}{l}\text { Dra. Eosina } \\
\text { @DraEosina }\end{array}$ & 29 \\
\hline & $\begin{array}{l}\text { Rodrigo Ugalde Herrá } \\
\text { @twiterrodri }\end{array}$ & 29 \\
\hline
\end{tabular}

Top 10 - Actividad

\begin{tabular}{|c|c|c|}
\hline pantit & & Tweets \\
\hline & $\begin{array}{l}\text { MD Ruben Garcia } \\
@ \text { @D_RDGA }\end{array}$ & 692 \\
\hline SEAP & $\begin{array}{l}\text { SEAP-IAP } \\
\text { @SEAP_IAP }\end{array}$ & 588 \\
\hline & $\begin{array}{l}\text { Eduardo Alcaraz MD } \\
\text { @edusqo }\end{array}$ & 389 \\
\hline & $\begin{array}{l}\text { Lara Pijuan,MD PhD } \\
\text { @lara_pijuan }\end{array}$ & 374 \\
\hline & $\begin{array}{l}\text { Jerad Gardner, MD } \\
\text { @JMGGardnerMD }\end{array}$ & 188 \\
\hline & $\begin{array}{l}\text { Dra. Eosina } \\
\text { @DraEosina }\end{array}$ & 160 \\
\hline & $\begin{array}{l}\text { Tania Labiano } \\
\text { @tlabiano }\end{array}$ & 158 \\
\hline & $\begin{array}{l}\text { Xelo Gimeno Borràs } \\
\text { @xelogimeno }\end{array}$ & 140 \\
\hline & $\begin{array}{l}\text { KALYANI BAMBAL } \\
\text { @kriyer68 }\end{array}$ & 109 \\
\hline & $\begin{array}{l}\text { Stefania Landolfi } \\
\text { @steland011 }\end{array}$ & 109 \\
\hline
\end{tabular}

Top 10 - Impresiones

Impresione

\begin{tabular}{|c|c|c|}
\hline & $\begin{array}{l}\text { Jerad Gardner, MD } \\
\text { @JMGardnerMD }\end{array}$ & $2,1 \mathrm{M}$ \\
\hline \multirow[t]{4}{*}{ SEAP } & $\begin{array}{l}\text { SEAP-IAP } \\
\text { @SEAP_IAP }\end{array}$ & $911,9 \mathrm{~K}$ \\
\hline & $\begin{array}{l}\text { Lara Pijuan,MD PhD } \\
\text { @lara_pijuan }\end{array}$ & $662,3 \mathrm{~K}$ \\
\hline & $\begin{array}{l}\text { Eduardo Alcaraz MD } \\
\text { @edusqo }\end{array}$ & $504,9 \mathrm{~K}$ \\
\hline & $\begin{array}{l}\text { Dra. Eosina } \\
\text { @DraEosina }\end{array}$ & $337,6 \mathrm{~K}$ \\
\hline & $\begin{array}{l}\text { diariomedico } \\
\text { @diariomedico }\end{array}$ & $303,1 \mathrm{~K}$ \\
\hline & $\begin{array}{l}\text { KALYANI BAMBAL } \\
\text { @kriyer68 }\end{array}$ & $285,2 \mathrm{~K}$ \\
\hline & $\begin{array}{l}\text { Pathology } \\
@ \text { @mvmd0908 }\end{array}$ & $258,1 \mathrm{~K}$ \\
\hline & $\begin{array}{l}\text { MD Ruben Garcia } \\
@ M D \_R D G A\end{array}$ & $254,6 \mathrm{~K}$ \\
\hline & $\begin{array}{l}\text { Xelo Gimeno Borràs } \\
\text { @xelogimeno }\end{array}$ & $177,8 \mathrm{~K}$ \\
\hline
\end{tabular}

Top 10 - IWantYouForSEAP

\section{Figura 5.}

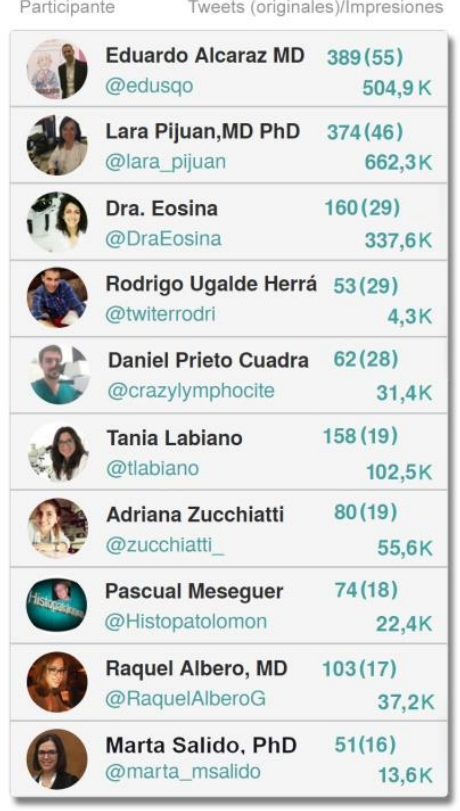

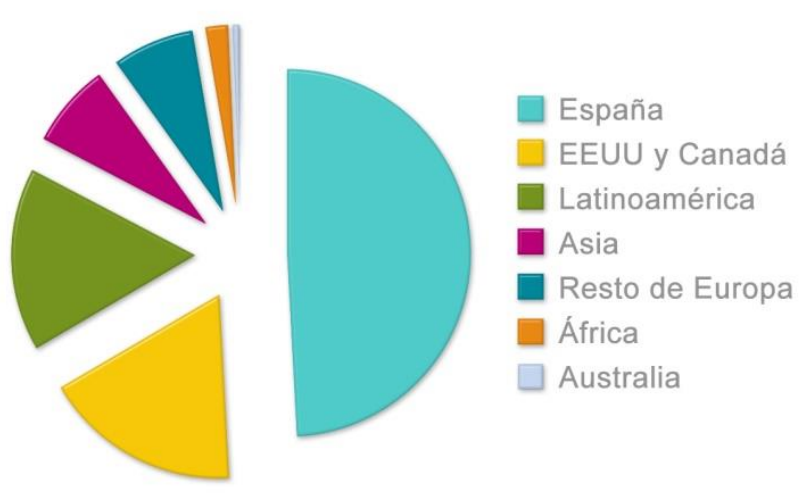

Figura 6. 